\title{
ACHIRAL 1-CUSPED HYPERBOLIC 3-MANIFOLDS NOT COMING FROM AMPHICHEIRAL NULL-HOMOLOGOUS KNOT COMPLEMENTS
}

\author{
KAZUHIRO ICHIHARA, IN DAE JONG, AND KOUKI TANIYAMA
}

\begin{abstract}
It is experimentally known that achiral hyperbolic 3-manifolds are quite sporadic at least among those with small volume, while we can find plenty of them as amphicheiral knot complements in the 3-sphere. In this paper, we show that there exist infinitely many achiral 1-cusped hyperbolic 3-manifolds not homeomorphic to any amphicheiral null-homologous knot complement in any closed achiral 3-manifold.
\end{abstract}

\section{INTRODUCTION}

An oriented 3-manifold is said to be achira 11 if it admits an orientation-reversing self-homeomorphism. As pointed out in [9, it is experimentally known that achiral hyperbolic 3-manifolds are quite sporadic at least among those with small volume. Similarly, the achiral cusped hyperbolic 3-manifolds obtained by Dehn fillings on the "magic manifold" are quite rare; just the figure-eight knot complement, its sibling, and the complement of the two-bridge link $S(10,3)$ [9, Proposition 2.9]. For the definition of a Dehn filling, see Section 3 ,

Among them, the figure-eight sibling ${ }^{2}$ is much more special. Precisely, the figureeight sibling admits an orientation-reversing self-homeomorphism $h$ for which there exists a slope $\gamma$ on a horotorus such that the distance between $\gamma$ and its image under $h$ is just one. Here a slope is an isotopy class of a non-trivial simple closed curve on a torus, and the distance of two slopes is defined as the minimal intersection number between their representatives.

We note that such a phenomenon does not occur for any amphicheiral nullhomologous knot complement. Here a knot $K$ in an achiral 3-manifold $M$ with an orientation-reversing self-homeomorphism $\varphi$ is said to be amphicheiral (with respect to $\varphi$ ) if $K$ is isotopic to $\varphi(K)$ in $M$. For an amphicheiral null-homologous knot $K$, with respect to the meridian-preferred longitude system for $K$, the slope $p / q$

2010 Mathematics Subject Classification. Primary 57M25; Secondary 57M50, 57N10.

Key words and phrases. Amphicheiral knot, banding, achiral hyperbolic 3-manifold, chirally cosmetic filling, chirally cosmetic surgery.

Ichihara is partially supported by Grant-in-Aid for Scientific Research (C) (No. 26400100), Japan Society for the Promotion of Science.

Taniyama is partially supported by Grant-in-Aid for Challenging Exploratory Research (No. 15K13439) and Grant-in-Aid for Scientific Research (A) (No. 16H02145), Japan Society for the Promotion of Science.

${ }^{1}$ The term "amphicheiral" is also used for the same notion on a 3-manifold. In this paper, we use the term "achiral" for a 3-manifold to clearly distinguish from an "amphicheiral knot".

${ }^{2}$ It is a unique complete orientable hyperbolic 3-manifold constructed by gluing together two ideal regular tetrahedra such that it is not homeomorphic to the figure-eight knot complement [14]. 
changed to $-p / q$ by the restriction of $\varphi$ to the complement of $K$ since $\varphi$ preserves the meridian-preferred longitude system for $K$. Note that the distance between the slopes $p / q$ and $-p / q$ is $2|p q|$, in particular, it is even. In addition, if $|p|,|q| \neq 0$, then the distance is greater than one.

In view of this, it is natural to ask if there exist achiral 3-manifolds not coming from any amphicheiral null-homologous knot complements (other than the figureeight sibling). As the main result in this paper, the following ensures that such 3 -manifolds surely exist.

Theorem 4.2. There exist infinitely many achiral 1-cusped hyperbolic 3-manifolds each of which is not homeomorphic to any amphicheiral null-homologous knot complement in any closed achiral 3-manifold.

One of our motivations to study such 3-manifolds comes from the research about cosmetic surgeries on knots in 4. There the achirality of the figure-eight sibling can be observed, and the 3-manifolds in Theorem 4.2 are considered as extensions to it. In fact, the first two authors asked the following in [4, Question 4.1].

Question 1.1. Can we find chirally cosmetic Dehn fillings on an achiral cusped hyperbolic 3-manifold along distance one slopes (other than the figure-eight sibling)?

The 3-manifolds in Theorem 4.2 give an affirmative answer to the above question as follows.

Corollary 4.7. There exist infinitely many achiral 1-cusped hyperbolic 3-manifolds each of which admits chirally cosmetic Dehn fillings along distance one slopes.

We construct our 3-manifolds in Section 2, which are given as the double branched cover of certain 2-string tangles. Then, after recalling the definitions of a Dehn surgery, a Dehn filling, a banding, and the Montesinos trick in Section 3 , we show in Section 4 that the interior of the obtained 3-manifolds are hyperbolic by using the Montesinos trick. In the last section, we show that each of our 3-manifolds is realized as the exterior of an amphicheiral knot in some achiral 3-manifold.

\section{EXAMPLES}

In this section, we construct achiral 3-manifolds by taking the double branched covers over certain "amphicheiral" 2-string tangles. Here, by a 2-string tangle or simply a tangle, we mean a pair consisting of a 3-ball and two properly embedded arcs in the 3 -ball.

Let $n$ be an integer with $n \neq 0,1$. Unless otherwise noted, we assume that $n \neq 0,1$ throughout this paper. Let us consider the tangle $T_{n}$ depicted in Figure 1 Here the box labelled $n$ (resp. $-n$ ) indicates $n$ times right-handed (resp. left-handed) half twists (see Figure 2). The construction of $T_{n}$ is based on the two-component link proposed by Nikkuni and the third author in [13, Figure 1.2].

Let $M_{n}$ be the compact oriented 3-manifold with a torus boundary obtained as the double branched cover over $T_{n}$. Then we have the following.

Proposition 2.1. The 3-manifold $M_{n}$ is achiral, and is not homeomorphic to any amphicheiral null-homologous knot exterior in any closed achiral 3-manifold.

Here for a knot $K$ in a 3-manifold $M$, we denote by $N(K)$ an open tubular neighborhood of $K$, and the exterior, denoted by $E(K)$, of $K$ is defined as $M \backslash N(K)$. Note that the interior of $E(K)$ is homeomorphic to the complement, $M \backslash K$, of $K$. 


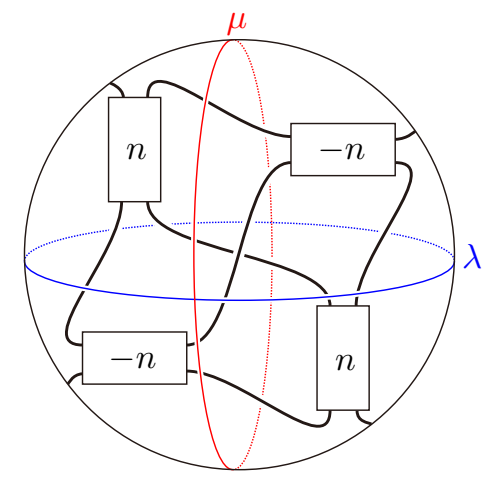

FiguRE 1 . The tangle $T_{n}$.

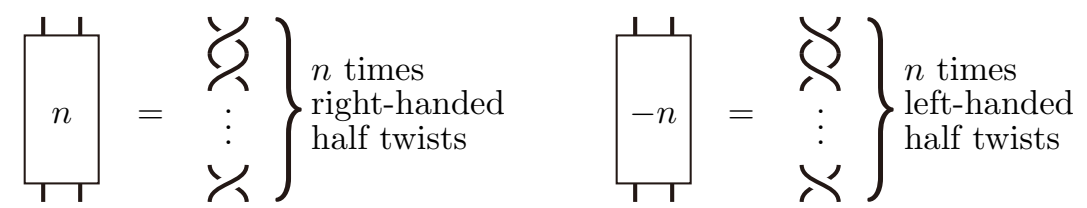

Figure 2. The cases where $n$ is positive (left-hand side) and negative (right-hand side).

Proof of Proposition 2.1. Let $m$ be the map mirroring $T_{n}$ along the plane of the paper, and let $T_{n} !=m\left(T_{n}\right)$. Notice that $T_{n}$ ! is the tangle obtained from $T_{n}$ by performing crossing changes at all the crossings. Let $M_{n}$ ! be the oriented 3-manifold obtained as the double branched cover over $T_{n}$ !. Then the lift $\widetilde{m}: M_{n} \rightarrow M_{n}$ ! is an orientation-reversing homeomorphism. On the other hand, since $T_{n}$ is obtained by a $\pi / 2$-rotation from $T_{n}$ !, $M_{n}$ ! is orientation-preservingly homeomorphic to $M_{n}$. Therefore $M_{n}$ admits an orientation-reversing self-homeomorphism $h=\widetilde{r} \circ \widetilde{m}$, where $\tilde{r}: M_{n} ! \rightarrow M_{n}$ is the lift of the $\pi / 2$-rotation $r: T_{n} ! \rightarrow T_{n}$. This implies that $M_{n}$ is achiral.

On the torus boundary $\partial M_{n}$ of $M_{n}$, we consider the two slopes $\widetilde{\mu}$ and $\widetilde{\lambda}$ appearing as the preimage of the two loops $\mu$ and $\lambda$ depicted in Figure 1 respectively. Then the distance between $\widetilde{\mu}$ and $\widetilde{\lambda}$ is just one. Since $r \circ m(\mu)=\lambda$, we have $h(\widetilde{\mu})=\widetilde{\lambda}$. Then, as explained in Section 1. $M_{n}$ is not homeomorphic to any amphicheiral null-homologous knot exterior in any closed achiral 3-manifold.

\section{Dehn surgery, BAnding, And Montesinos trick}

In this section, we give a review on a Dehn surgery, a Dehn filling, a banding, and the Montesinos trick, to show that the interior of $M_{n}$ is hyperbolic for $n \neq 0,1$.

3.1. Dehn surgery. Let $K$ be a knot in a closed oriented 3-manifold $M$ with the exterior $E(K)$. Let $\gamma$ be a slope on the boundary torus $\partial E(K)$. Then, the Dehn surgery on $K$ along $\gamma$ is defined as the following operation: Glue a solid torus $V$ to $E(K)$ such that a simple closed curve representing $\gamma$ bounds a meridian disk in $V$. We denote by $K(\gamma)$ the obtained 3-manifold. It is said that the Dehn surgery along the meridional slope is the trivial Dehn surgery. Also it is said that a Dehn 
surgery along a slope which is represented by a simple closed curve intersecting the meridian at a single point is an integral Dehn surgery.

In the case where $K$ is null-homologous in $M$, we have the well-known bijective correspondence between $\mathbb{Q} \cup\{1 / 0\}$ and the set of slopes on $\partial E(K)$, which is given by using the meridian-preferred longitude system for $K$. When the slope $\gamma$ corresponds to $r \in \mathbb{Q} \cup\{1 / 0\}$, then the Dehn surgery on $K$ along $\gamma$ is said to be the $r$-surgery on $K$, and the obtained 3-manifold is denoted by $K(r)$. In this case, an integral Dehn surgery corresponds to an $n$-surgery with an integer $n$.

Two Dehn surgeries on a knot $K$ along two slopes are said to be chirally cosmeti 3 if two obtained 3-manifolds are orientation-reversingly homeomorphic.

3.2. Dehn filling. While there are some overlaps with notions on Dehn surgery, here we briefly review a Dehn filling. Let $M$ be a compact connected oriented 3manifold with a torus boundary $\partial M$, and $\gamma$ a slope on $\partial M$. The Dehn filling on $M$ along $\gamma$ is the operation gluing a solid torus $V$ to $M$ so that a simple closed curve representing $\gamma$ bounds a meridian disk in $V$. As in the case for a Dehn surgery, if we have the correspondence between the slope $\gamma$ and $r \in \mathbb{Q} \cup\{1 / 0\}$ using the meridian-preferred longitude system, then the Dehn filling on $M$ along $\gamma$ is said to be the $r$-Dehn filling on $M$.

Two Dehn fillings on $M$ along two slopes are said to be chirally cosmetic if the resultants are orientation-reversingly homeomorphic. For recent studies on chirally cosmetic Dehn fillings, we refer the reader to [4, 5, 6, 12.

3.3. Banding. We call the following operation on a link a banding ${ }^{4}$ on the link. For a given link $L$ in $S^{3}$ and an embedding $b: I \times I \rightarrow S^{3}$ such that $b(I \times I) \cap L=$ $b(I \times \partial I)$, where $I$ denotes a closed interval, we obtain a (new) link as $(L-b(I \times$ $\partial I)) \cup b(\partial I \times I)$.

Remark 3.1. On performing a banding, it is often assumed the compatibility of orientations of the original link and the obtained link, but in this paper, we do not assume that. Also note that this operation for a knot yielding a knot appears as the $n=2$ case of the $H(n)$-move on a knot, which was introduced in 3 .

It is well-known that a rational tangle is determined by the meridional disk in the tangle. The boundary curve of the meridional disk is parameterized by an element of $\mathbb{Q} \cup\{1 / 0\}$, called a slope of the rational tangle. A rational tangle is said to be integral if the slope is an integer or $1 / 0$. For brevity, we call an integral tangle with a slope $n$ an $n$-tangle.

A banding can be regarded as an operation replacing a 1/0-tangle into an $n$ tangle. Then we call this banding an $n$-banding.

A banding on a link $L$ is said to be chirally cosmetic if the link obtained from $L$ by the banding is ambient isotopic to the mirror image $L$ ! of $L$ in $S^{3}$.

3.4. Montesinos trick. We here recall the Montesinos trick originally introduced in 11. Let $\Sigma$ be the double branched cover of $S^{3}$ branched along a link $L \subset S^{3}$. Let $K$ be a knot in $\Sigma$, which is strongly invertible with respect to the preimage $\bar{L}$ of $L$, that is, there is an orientation preserving involution of $\Sigma$ with the quotient $S^{3}$ and

\footnotetext{
${ }^{3}$ This terminology was used in Kirby's problem list [8. In [2], it is called reflectively cosmetic.

${ }^{4}$ The operation is sometimes called a band surgery, a bund sum (operation), or a hyperbolic transformation in a variety of contexts. In this paper, referring to [1, we use the term banding to clearly distinguish it from a Dehn surgery on a knot.
} 
the fixed point set $\bar{L}$ which induces an involution of $K$ with two fixed points. Then the 3-manifold $K(\gamma)$ obtained by an integral Dehn surgery on $K$ is homeomorphic to the double branched cover $\Sigma^{\prime}$ along the link $L^{\prime}$ obtained from $L$ by a banding along the band appearing as the quotient of $K$. That is, we have the following commutative diagram:

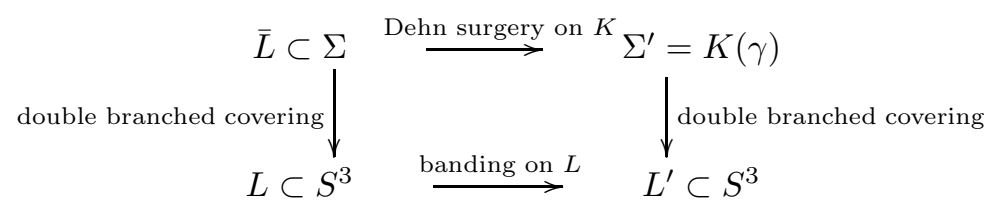

\section{Hyperbolicity}

In this section, we show that the interior of our 3-manifolds $M_{n}$ given in Section2 are hyperbolic, and prove our main theorem. Let $K_{n}$ be the link in $S^{3}$ obtained by closing the tangle $T_{n}$ as shown in Figure 3.

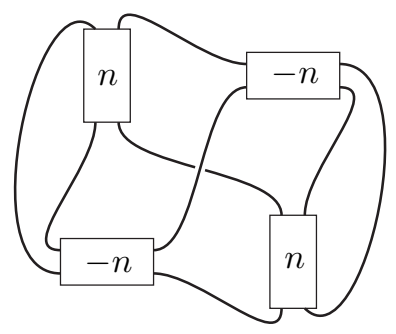

Figure 3 . The link $K_{n}$.

Proposition 4.1. The knot $K_{n}$ is the two-bridge knot (or link) with Schubert's normal form

$$
S\left(n^{4}-2 n^{3}+2 n^{2}-2 n+1, n^{3}-2 n^{2}+n-1\right) .
$$

For the definitions of Schubert's normal form and Conway's normal form for a two-bridge link, see for example [7, Section 2.1].

Proof of Proposition 4.1. One can diagrammatically check that $K_{n}$ is the twobridge knot (or link) with Conway's normal form $C(n, n,-1, n, n)$. Calculating the continued fraction, we have

$$
\frac{1}{n+\frac{1}{n+\frac{1}{-1+\frac{1}{n+\frac{1}{n}}}}}=\frac{n^{3}-2 n^{2}+n-1}{n^{4}-2 n^{3}+2 n^{2}-2 n+1} .
$$

We note that $K_{n}$ is a two-bridge knot when $n$ is even, and a two-bridge link when $n$ is odd. Also note that $K_{2}=S(5,1)$ is the $(2,5)$-torus knot.

As shown in Figure 4, each of $K_{n}$ admits a chirally cosmetic banding. In particular, for $K_{2}$, this chirally cosmetic banding was essentially discovered by Zeković [15, 


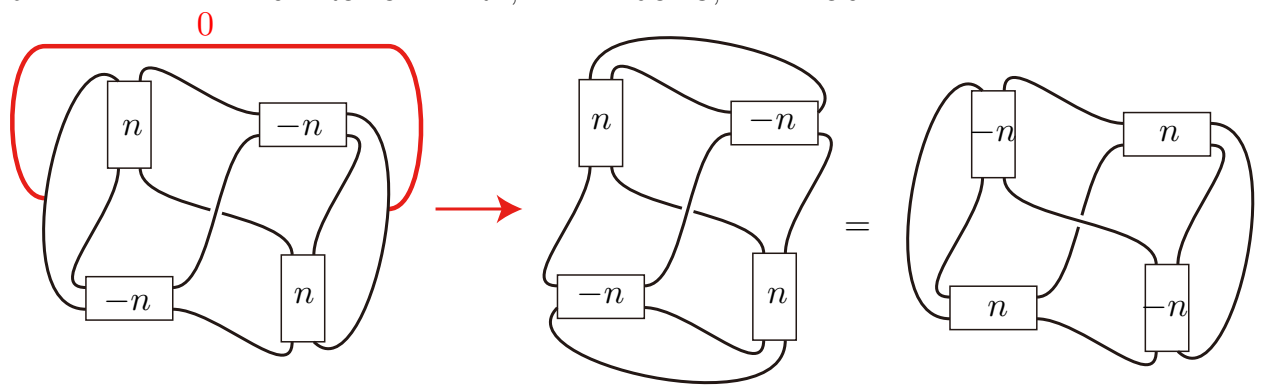

FiguRE 4 . The 0-banding on $K_{n}$ yields the mirror image $K_{n}$ !.

and pointed out by the first two authors that the upstairs of this banding corresponds to the chirally cosmetic filling on the figure-eight sibling, see [4, Section 4].

Let $\Sigma_{n}$ be the lens space of type $\left(n^{4}-2 n^{3}+2 n^{2}-2 n+1, n^{3}-2 n^{2}+n-1\right)$ which is obtained as the double branched cover of $S^{3}$ branched along $K_{n}$. Since $n \neq 0,1$, $\Sigma_{n}$ is not homeomorphic to $S^{3}$ or $S^{2} \times S^{1}$. Then we obtain the following.

Theorem 4.2. There exist infinitely many achiral 1-cusped hyperbolic 3-manifolds each of which is not homeomorphic to any amphicheiral null-homologous knot complement in any closed achiral 3-manifold.

Proof. Applying the isotopic deformation and the Montesinos trick conversely to the chirally cosmetic banding on $K_{n}$ shown in Figure 4 by Proposition 4.1 , we obtain a surgery descriptions of $\Sigma_{n}$ and $-\Sigma_{n}$ as in Figure 5 That is, the trivial Dehn surgery and the 0-surgery on the red component in Figure 5 yield $\Sigma_{n}$ and $-\Sigma_{n}$ respectively.

Drilling along the red component, we obtain the surgery description of the 3manifold with a torus boundary $M_{n}$ as in Figure 6, which is already constructed in Section 2. Then $M_{n}$ is the exterior of a knot in $\Sigma_{n}$, which admits chirally cosmetic Dehn fillings along distance one slopes.

By the classification of cosmetic surgeries on a non-hyperbolic knot in a lens space due to Matignon [10, we see that the interior of $M_{n}$ is hyperbolic. In fact, for a non-hyperbolic knot $J$ in a lens space other than $S^{3}$ or $S^{2} \times S^{1}$, if the trivial Dehn surgery and the $r$-surgery on $J$ are chirally cosmetic, then $r \neq 0$, see [10, Theorems 3.2 and 4.1].

It is enough to show that if $n \neq n^{\prime}$, then $M_{n}$ is not homeomorphic to $M_{n^{\prime}}$. Then the following lemma completes the proof.

Lemma 4.3. We have $H_{1}\left(M_{n} ; \mathbb{Z}\right) \cong \mathbb{Z} \oplus \mathbb{Z}_{n^{3}-n^{2}+n-1}$.

Proof. Let $X$ be the exterior of the 6 -components link in $S^{3}$ as in Figure 7 Taking representatives of meridians $x, a, b, c, d, e$ as in Figure 7 , we have

$$
H_{1}(X ; \mathbb{Z}) \cong \mathbb{Z}^{6}=\langle a\rangle \oplus\langle b\rangle \oplus\langle c\rangle \oplus\langle d\rangle \oplus\langle e\rangle \oplus\langle x\rangle
$$



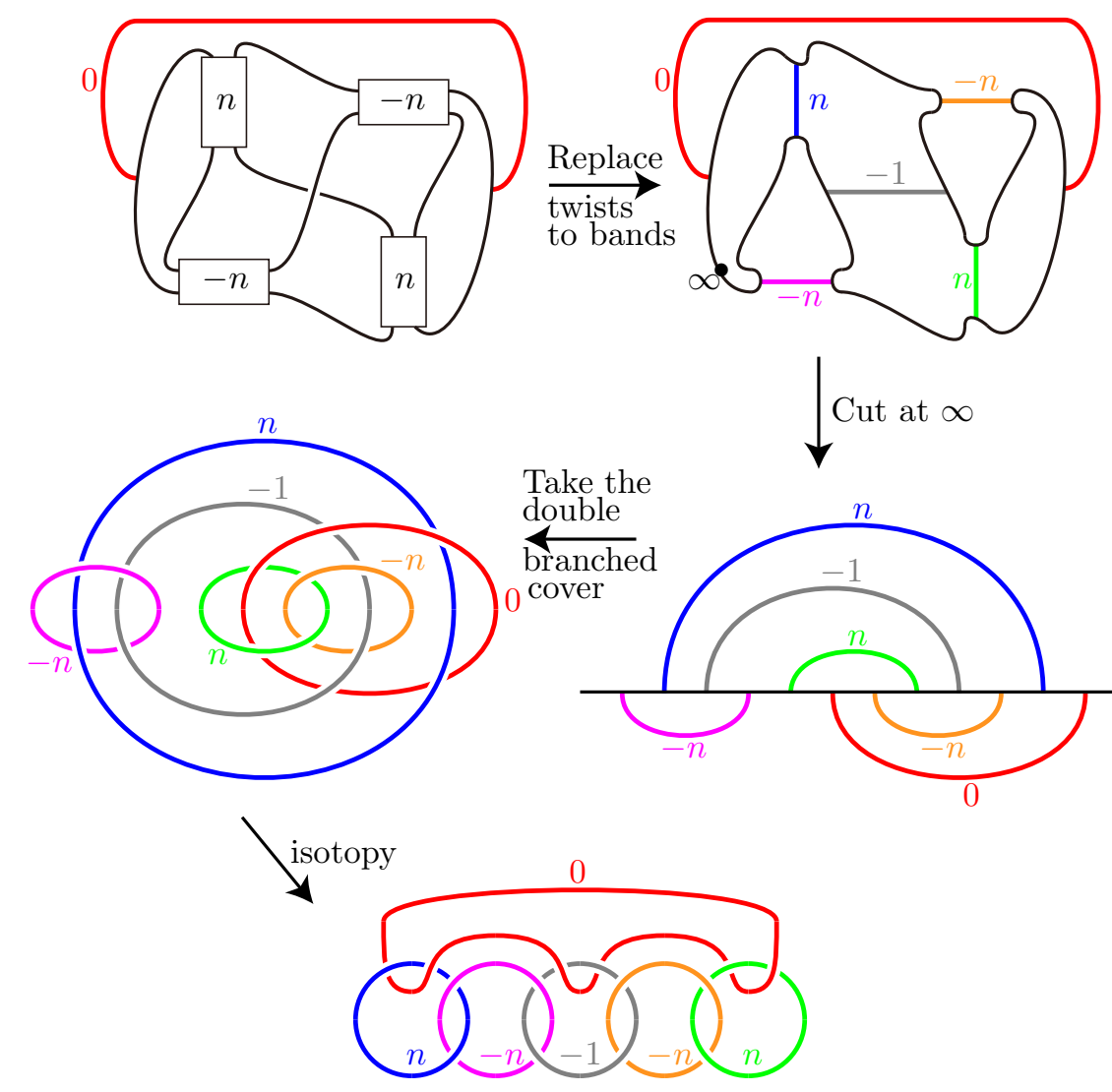

$\downarrow$ Cut at $\infty$

Take the

over

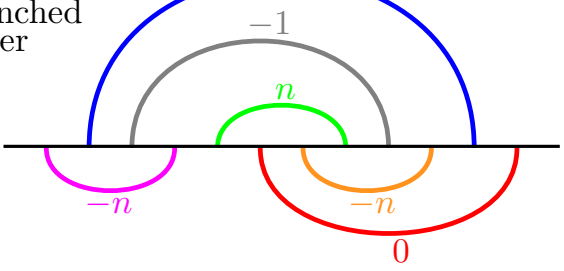

Figure 5. Applying the Montesinos trick.

Reading off the slopes from Figure [ we see that suitable Dehn fillings on the five boundaries supply the following relations.

$$
\left\{\begin{array}{l}
n a+x-b=0, \\
-n a+c-a=0, \\
-c+b-x-d=0, \\
-n d-c+e=0, \\
n e+d+x=0 .
\end{array}\right.
$$

Then we have

$$
\left(\begin{array}{cccccc}
n & -1 & 0 & 0 & 0 & 1 \\
-1 & n & 1 & 0 & 0 & 0 \\
0 & 1 & -1 & -1 & 0 & -1 \\
0 & 0 & -1 & -n & 1 & 0 \\
0 & 0 & 0 & 1 & n & 1
\end{array}\right)
$$

as the presentation matrix of $H_{1}\left(M_{n} ; \mathbb{Z}\right)$. Reducing the matrix by the elementary operations on presentation matrices of modules (for example see [7, Lemma 7.2.1]), we see that the previous matrix is equivalent to

$$
\left(n^{3}-n^{2}+n-1 \quad 0\right) \text {. }
$$


Thus we have $H_{1}\left(M_{n} ; \mathbb{Z}\right) \cong \mathbb{Z} \oplus \mathbb{Z}_{n^{3}-n^{2}+n-1}$.

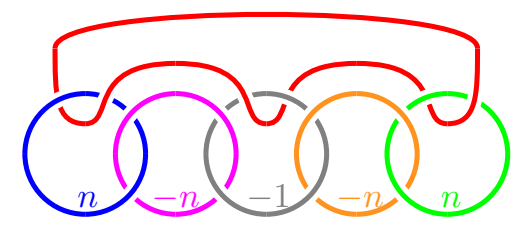

FiguRe 6 . A surgery description of $M_{n}$

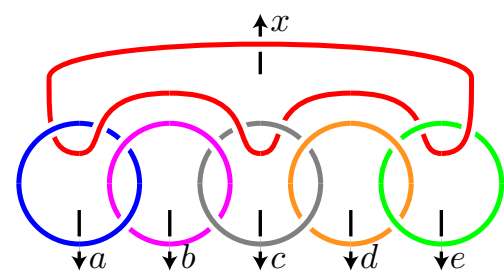

Figure 7 . Generators of $H_{1}(X ; \mathbb{Z})$.

Remark 4.4. By the classification of lens spaces, for example see [7, Theorem D.1.2], $\Sigma_{n}$ is chiral (i.e. not achiral) since we have

$$
\left(n^{3}-2 n^{2}+n-1\right)^{2} \equiv 1 \not \equiv-1 \quad \bmod \left(n^{4}-2 n^{3}+2 n^{2}-2 n+1\right) .
$$

Remark 4.5. For any null-homologous knot $J$ in an oriented closed 3-manifold $Y$, we have $H_{1}(Y \backslash J ; \mathbb{Z}) \cong \mathbb{Z} \oplus H_{1}(Y ; \mathbb{Z})$. Each of our 3 -manifolds $M_{n}$ is the exterior of a knot, say $J_{n}$, in the lens space $\Sigma_{n}$. By Lemma 4.3, one can also see that $J_{n}$ is not null-homologous in $\Sigma_{n}$ since $H_{1}\left(\Sigma_{n} ; \mathbb{Z}\right)=\mathbb{Z}_{n^{4}-2 n^{3}+2 n^{2}-2 n+1}$. In the case where $n=2$, we have $n^{4}-2 n^{3}+2 n^{2}-2 n+1=n^{3}-n^{2}+n-1=5$. This case corresponds to the chirally cosmetic fillings on the figure-eight sibling introduced in Section 1 . Thus, the knot $J_{2}$ is also not null-homologous in $\Sigma_{2}$.

Remark 4.6. As shown in Figure 8, we can see directly that the 0-surgery on the red component in Figure 6 changes $\Sigma_{n}$ to $-\Sigma_{n}$.

As in the proof of Theorem 4.2, $M_{n}$ admits chirally cosmetic Dehn fillings along distance one slopes. Thus, we have the following.

Corollary 4.7. There exist infinitely many achiral 1-cusped hyperbolic 3-manifolds each of which admits chirally cosmetic Dehn fillings along distance one slopes.

\section{REALizing $M_{n}$ AS AN AMPhicheiral KNOt COMPLEMENT}

In this section, we show the following.

Proposition 5.1. Each of our 3-manifolds $M_{n}$ can be realized as the exterior of an amphicheiral knot in some achiral 3-manifold.

Note that such an amphicheiral knot is not null-homologous. 

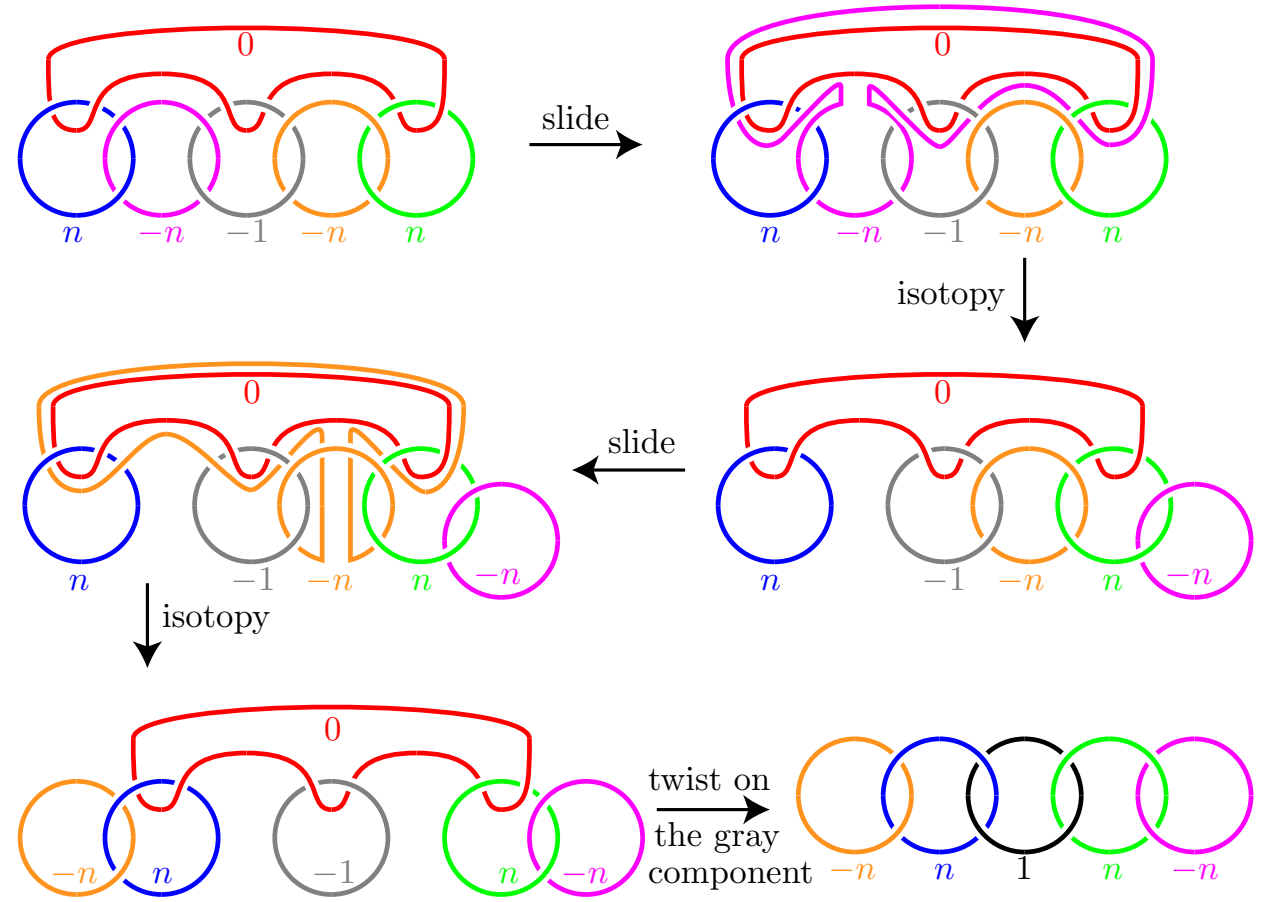

FiguRE 8 . The 0 -surgery changes $\Sigma_{n}$ to $-\Sigma_{n}$.

Proof of Proposition 5.1. As in Sections 2 and 4, the meridian on $\partial M_{n}$ is $\widetilde{\mu}$, and we can choose the longitude on $\partial M_{n}$ as $\tilde{\lambda}$ corresponding to $0 / 1$. With respect to this meridian-longitude system, we see that the two slopes corresponding to $1 / 1$ and $-1 / 1$ are invariant via the orientation-reversing self-homeomorphism $h=\widetilde{r} \circ \widetilde{m}$ on $M_{n}$, see Figure 9 .

Let $N_{n}^{ \pm}$be the closed oriented 3-manifold obtained from $M_{n}$ by $\pm 1 / 1$-Dehn filling respectively. Since the core curve $c$ of the attached solid torus $V$ intersects a meridian disk of $V$ once, $h$ extends to $V$, and thus, $h$ extends to $N_{n}^{ \pm}$. This implies that $N_{n}^{ \pm}$is achiral. Further, for the knot $k$ in $N_{n}^{ \pm}$, which corresponds to the core $c$, $k$ is an amphicheiral knot in $N_{n}^{ \pm}$and the exterior of $k$ is homeomorphic to $M_{n}$.
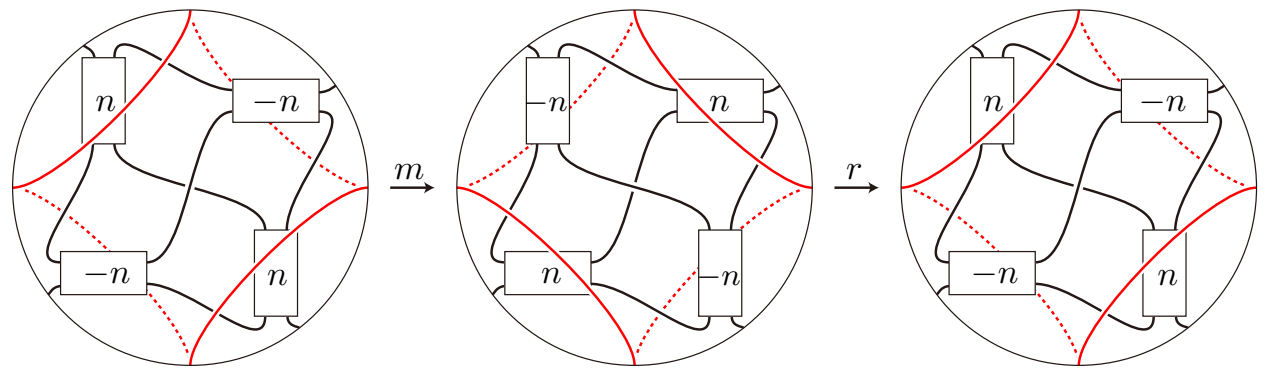

Figure 9. The red loop corresponding to the slope $1 / 1$ on $\partial T_{n}$ is invariant via $r \circ \mathrm{m}$. 
Acknowledgement. The authors would like to thank Professor Kimihiko Motegi and Professor Kai Ishihara for useful comments on Section 5

\section{REFERENCES}

[1] S. A. Bleiler, Banding, twisted ribbon knots, and producing reducible manifolds via Dehn surgery, Math. Ann. 286 (1990), no. 4, 679-696.

[2] S. A. Bleiler, C. D. Hodgson and J. R. Weeks, Cosmetic surgery on knots, in Proceedings of the Kirbyfest (Berkeley, CA, 1998), 23-34, Geom. Topol. Monogr., 2, Geom. Topol. Publ., Coventry.

[3] J. Hoste, Y. Nakanishi and K. Taniyama, Unknotting operations involving trivial tangles, Osaka J. Math. 27 (1990), no. 3, 555-566.

[4] K. Ichihara, I. D. Jong, and H. Masai, Cosmetic banding on knots and links, to appear in Osaka J. Math., arXiv:1602.01542.

[5] K. Ichihara and T. Saito, Cosmetic surgery and the $S L(2, \mathbb{C})$ Casson invariant for two-bridge knots, to appear in Hiroshima Math. J., arXiv:1602.02371

[6] K. Ichihara and Z. Wu, A note on Jones polynomial and cosmetic surgery, to appear in Comm. Anal. Geom., arXiv:1606.03372

[7] A. Kawauchi, A survey of knot theory, translated and revised from the 1990 Japanese original by the author, Birkhäuser Verlag, Basel, 1996.

[8] Problems in low-dimensional topology, in Geometric topology (Athens, GA, 1993), 35-473, AMS/IP Stud. Adv. Math., 2.2, Amer. Math. Soc., Providence, RI.

[9] B. Martelli and C. Petronio, Dehn filling of the "magic" 3-manifold, Comm. Anal. Geom. 14 (2006), no. 5, 969-1026.

[10] D. Matignon, On the knot complement problem for non-hyperbolic knots, Topology Appl. 157 (2010), no. $12,1900-1925$

[11] J. M. Montesinos, Surgery on links and double branched covers of $S^{3}$, in Knots, groups, and 3-manifolds (Papers dedicated to the memory of R. H. Fox), 227-259. Ann. of Math. Studies, 84, Princeton Univ. Press, Princeton, NJ.

[12] Y. Ni and Z. Wu, Cosmetic surgeries on knots in $S^{3}$, J. Reine Angew. Math. 706 (2015), $1-17$.

[13] R. Nikkuni and K. Taniyama, Symmetries of spatial graphs and Simon invariants, Fund. Math., 205 (2009), no. 3, 219-236.

[14] J. Weeks, Hyperbolic structures on three-manifolds, PhD thesis, Princeton University, 1985.

[15] A. Zeković, Computation of Gordian distances and $\mathrm{H}_{2}$-Gordian distances of knots, Yugosl. J. Oper. Res. 25 (2015), no. 1, 133-152.

Department of Mathematics, College of Humanities and Sciences, Nihon University, 3-25-40 Sakurajosui, Setagaya-ku, Tokyo 156-8550, Japan.

E-mail address: ichihara@math.chs.nihon-u.ac.jp

Department of Mathematics, Kindai University, 3-4-1 Kowakae, Higashiosaka City, OSAKA 577-0818, JAPAN

E-mail address: jong@math.kindai.ac.jp

Department of Mathematics, School of Education, Waseda University, Nishi-Waseda 1-6-1, SHINJUKU-KU, TOKYO, 169-8050, JAPAN

E-mail address: taniyama@waseda.jp 